\title{
Socially-aware emergent narrative
}

\author{
Sergio Alvarez-Napagao, Ignasi Gómez-Sebastià, Sofia Panagiotidi, Arturo \\ Tejeda-Gómez, Luis Oliva, and Javier Vázquez-Salceda \\ Universitat Politècnica de Catalunya \\ \{salvarez, igomez, panagiotidi, jatejeda, loliva, jvazquez\}@lsi.upc.edu
}

\begin{abstract}
In agent research, emergent narrative aims for practical solutions to the narrative paradox problem in both drama and interactive scenarios. At the same time, organisational frameworks can be used in games to provide flexibility, adaptiveness, or social-awareness. In this paper, we propose an extension of our CONCIENS framework to support emergent narrative in games with two objectives: 1) provide socialawareness in emergent narrative by means of an organisational model, and 2) create convincing dynamic and flexible storytelling in games.[?]
\end{abstract}

\section{Introduction}

The main objective of the use of Artificial Intelligence (AI) in both fun and serious games is to deliver the illusion of "intelligence" in the non-player characters' (NPCs) behaviour. While some aspects - e.g., pathfinding - have evolved to a mature state in both the industry and academic research, it is not the case with some important ones such as individual behaviour or strategical reasoning.

Current challenges deal with high-level concepts of gaming such as realistic virtual actors, automatic content and storyline generation, dynamic learning, or social behavior. Tackling these issues could represent a qualitative improvement on gaming experience from the player perspective and academic research on AI has good opportunities to provide solutions to these challenges [10.

Solutions taken by the industry are mainly based on domain-dependent lowlevel approaches. These solutions arise some obvious issues [3]: lack of flexibility and adaptation to environmental change, predictable or strange behaviour, low reusability, or blind specifications of NPCs - i.e. the NPCs always know how to act, few times they know what they are doing, but very rarely they know why.

One important factor that leads to these problems is the need for a plot or storyline. NPCs are usually mere enactors of a story previously designed, and their main use is to help advancing the story rather than acting on their own. It is well known that there is a compromise between narrative control and character autonomy [11. This has been a topic of interest from the agent community in what has usually been called emergent narrative: stories can emerge through simulation of a virtual world inhabited by virtual characters.

As a result of research on emergent narrative, some theoretical frameworks and implementations have appeared, focusing on both plot and characters. In this paper we add a social aspect to this formula by linking our previous work on organisational frameworks for games. 


\section{Emergent narrative}

Emergent narrative tries to break the common conception of linear narrative being the only possible product of human authorship over a story: human authorship can also be applied to the creation of a more open narrative by balancing character models, event sequences, and narrative landmarks. Furthermore, research on this topic tries to tackle the problem of the narrative paradox: virtual environments - such as games - and narratives exist on different ontological levels, and thus there is a fundamental conflict between free-form interactivity provided by the virtual environment and the level of satisfaction produced by a man-made narrative structure 11. The main hypothesis of emergent narrative is that this problem cannot be solved by treating both issues as separate and combining them, but by treating narrative as a direct result of the actions of the characters [5].

In FearNot! and its agent architecture FAtiMA [6], narrative control is achieved by organising the story in episodes at design-time and sequencing them at runtime. Each episode defines pre- and post-conditions, as well as sets of possible locations, objects, choices, and goals available. However, as discussed in 11, such a strong episodic design is limiting, as a global sense of time - and what happens during scenes, or what happens between them in the "world"- or emotional residue after each scene are not accounted for.

One way to reduce rigidness in narrative control is distributed drama management, combined with double appraisal 9 . The main idea is that characters take responsibility in managing the drama, including in their plan selection mechanism a bias towards choices that have the greatest impact on the emotions of other characters. This idea of distributed drama management has been adopted by the Virtual Storyteller [11, the architecture of which is depicted in Figure 1. Character agents are based on the FAtiMA agent architecture and the world agent is the interface to a simulation layer. The plot agent acts as an intermediary, setting up the simulation and sending perceptions to, and receiving actions from the character agents. Stories are stored using Fabula [11, a formal model based on causal network theory to represent events already occurred with respect to the story. Events are linked to other narrative concepts such as goals, actions, or perceptions, via causal relationships - e.g., physical or psychological. The resultant graph is then used by both the presentation and simulation layers, and can be used for further analysis.

In this framework, characters enact two highly coupled roles: in-character (IC) and out-of-character (OOC) 4]. The former refers to the character behaviour and is driven by individual motivations, as normal agents. The latter, however, constrains the behaviour by trying to increase narrative impact - e.g., adopting goals that will probably cause conflict with other characters, looking for a modification on the relationship with them, or making sure that there are always goals to pursue. The action pursued by a story character will then be a function [1] upon believability (IC role), dramatic opportunity and variability (OOC role). 


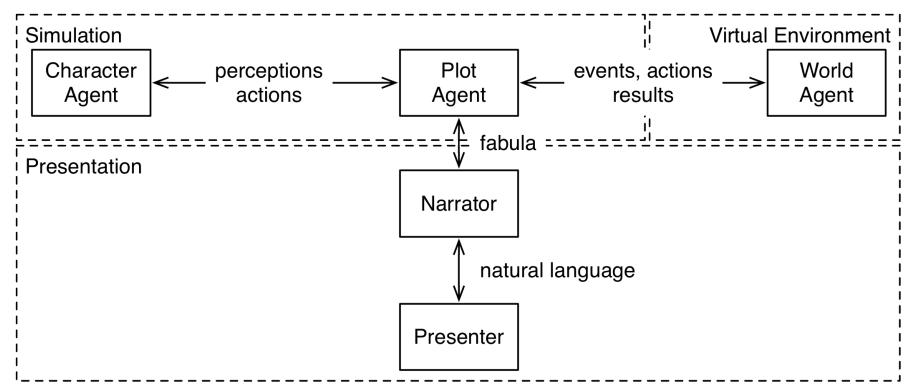

Fig. 1. Virtual Storyteller architecture

In Virtual Storyteller, emergent narrative is achieved by influencing the event sequence in order to create choices for the IC role while giving more chances to achieve the OOC role. This can be done in two ways, taken from drama improvisation techniques. Making events happen consists in creating an event that will likely enforce an advancement in the plot, e.g., the Princess has been kidnapped by a dragon, thus forcing those characters looking for brave actions to go and save her. Late commitment is based on the assumption that parts of the initial state of the world do not need to be fixed at authoring time, but dynamically determined at run time when it is purposeful for narrative purposes. In late commitment, OOC roles look for feasible and consistent properties to be added to the initial state and which will provide opportunities to advance the plot towards the storyline objectives, e.g., the story could advance by suddenly discovering that the governor is, in fact, a spy of the enemy.

Although the Virtual Storyteller presents a sound architecture for emergent narrative, it is strongly focused on non-interactive storytelling. This has already been noted in [1], stating that games allow for more radical applications of narrative control techniques such as late commitment. Also, from our point of view, the social aspect of multi-agent systems is somehow ignored by keeping character agents as a separate component from the simulation layer.

\section{Organizational frameworks and games}

As discussed on 3], our hypothesis is that it is possible to create elaborate solutions for the issues of both individual behavior control and collective strategy techniques by integrating models based on Organization Theoretical methods to control NPCs' behavior. This theory contributes to the systematic study of how actors behave within organizations. Hence, the actors in a game are described as an organization the behaviour of which is based on specific roles, norms, dependencies, and capabilities.

In fact, organizational frameworks such as OperA are already being explored for their use in serious games. In [12, organizational specifications are used to create a distributed intelligent task selection system that adapts to the player skill level and models the storyline. 
CONCIENS [2] advances on this line of work by generalizing the use of organizational models for fun games, more focused on the realism of gaming experience, rather than on user modeling and learning. CONCIENS adapts the ALIVE framework [1 to its use in games and allows Game AI developers to think in terms of why-what-how when defining the decision-making actions for NPCs. That is, at the Organizational level, the developer defines "why to do something" by describing the elements of the organizational structure in terms of organization objectives, roles, norms, and restrictions. At the Coordination level, the developer defines "what to do" based on possible solutions and tasks to realize in specific situations; finally, at the Game Enacting level, the developer defines "how to do it" in terms of which actual, game-specific actions to perform in order to realize those tasks.

The set of tools and methods of CONCIENS provides inherent support to the development of complex, re-usable Game AI solutions, extending the ALIVE environment by providing:

1. A practical solution to couple agents to the Game Engine, by defining the Game Enactor programming interface.

2. A tool to describe the Organization Ontology, which contains a representation of agent structures.

3. The elements to describe game actors' behavior via social structures based on norms, roles and their enactment, promoting the balance between autonomy and story direction.

The research aim of CONCIENS is to provide solutions to the issues presented in Section 1 by representing the interactions between players and NPCs as compliant to an organisational structure. This approach provides extended flexibility to the elements that imply intelligent behavior, e.g. actors and characters, teams of individuals, and narrative storylines. In addition, it provides a methodology and metrics that can be applied to evaluate the organizational behavior using the games' environments as simulation scenarios. Hence, it would be possible to compare, learn, and improve NPC's behavior with an approach based on organization theoretical solutions for Game AI, contributing to overall flexibility and adaptiveness.

CONCIENS has already been used to implement automatic and flexible team direction in real-time strategy games [2, and to showcase an improved method to detect and enforce traffic violations in free roaming games [3]. The next goal in our research is to test adaptive storytelling in multiplayer games by using narrative emergence, and we will show in Section 4 how we intend to achieve it.

\section{Our proposal}

In this section we present our proposal, an extension of CONCIENS to adopt the architecture and some mechanisms of Virtual Storyteller to enable emergent narrative in games. 


\subsection{Mapping Virtual Storyteller components to cOncienS}

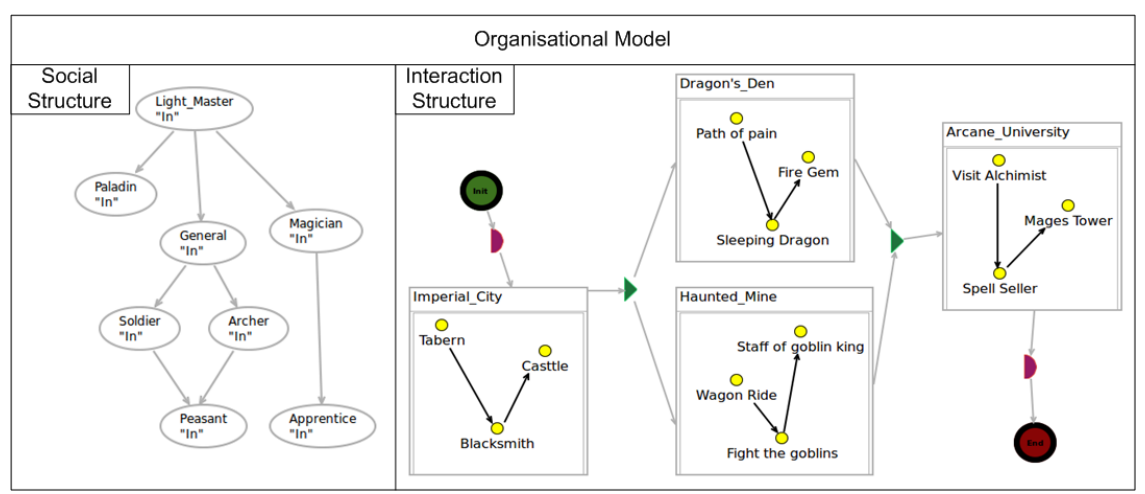

Fig. 2. Organisational Model Example

In CONCIENS, everything starts from the organisational description (Figure 21), instanced as OperA documents. OperA consists of two main components, the Social Structure and the Interaction Structure. The Social Structure assigns roles to human players based on their preferences, and can be adapted to meet player's needs, for instance, Apprentice role can be removed if there is no player willing to play it. The Interaction Structure shows a set of scenes important to the overall plot. Each scene contains a set of landmarks that are important states of the world regarding the scene. Both scenes and landmarks are connected via transition arcs that allow navigating through them. Therefore, agents representing NPCs and players, by using these organisational constructs, become social-aware: they will be able to reason about their relationship with each other in terms of joint objectives, social rules and common interaction patterns.

We intend to incorporate the components of the Virtual Storyteller (see Figure 1) as an adaptation of the CONCIENS framework as depicted in Figure 3. The components that enable emergent narrative are: the Character Agents, which support both NPCs and players and are represented in CONCIENS by the already existing agents of the agent layer; the World Agent, represented by the Global Monitor; the Narrator layer, implemented by the Game Enactor - i.e., converting the world state into generic game concepts such as movement orders or player quests -; and the Presenter layer, in our case the Game Engine. The only new component required is the Plot Agent.

\subsection{Constructing the plot}

The Plot Agent will receive a storyline from the story designer. This storyline is implemented as a set of scenes and landmark patterns: the minimal set of states that conform the story and that have to be fulfilled in its proper order, from the 
beginning to the end of the gameplay time. This agent will continuously observe the state of the world and dynamically plan an order of the scenes needed to get to the next storyline landmark. The roles in each scene are assigned to specific Character Agents.

The story designer can decide, in this way, how rigid / flexible the story should be by adding more or less landmarks, and by declaring stronger or weaker conditions as landmarks. The designer will also design the set of possible late commitments and non-causal related events (see Section 2) in the form of framing operators [11, that is, sets of preconditions and a set of effects on these operators that can be done if the change in a specific case is consistent with the history of events - by the use of Fabula [11].

\subsection{Character conflicts and personality}

The Character Agent is a BDI agent implemented inside each agent of the CONCIEnS agent level. Every NPC, as well as every player, has a representation as a Character Agent. The IC role (as seen in Section 2) is already implemented at the CONCIENS framework. Egoistic motivations, aims, capabilities, individual behaviour and organisational constraints (social objectives and norms acting as constraints to its behaviour or capabilities) are taken into account by the agent in an autonomous decision making process that produces an appropriate plan. This plan fulfills the agent's personal specifications bringing its own ways into the organisational society as well.

On the other hand, as seen earlier, the agent receives from the Plot Agent a set of landmarks that is processed by

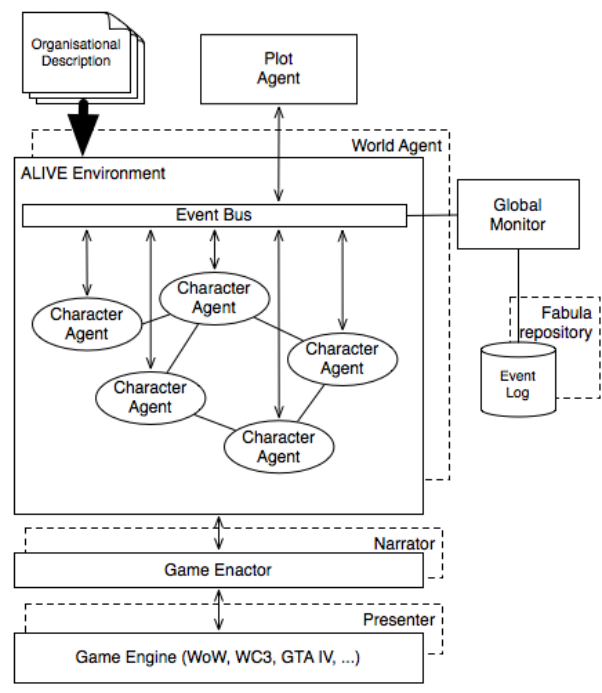

Fig. 3. Adapted COnCIEnS architecture the OOC role to help advancing the story. Due to this dual nature of the Character Agents, conflicts between the IC and the OOC can - and probably will - arise. This can be solved by applying negotiation processes, such as argumentation, and will be one of the main focuses of research on this project.

In order to apply personality to the characters - including players -, we will characterise them by using stereotypes or play styles. There are two main taxonomies to identify play styles: DGD1 [7] (Conqueror, Manager, Wanderer, Participant), and interaction between players: Interest Model [8] (Achievers, Explorers, Socialisers, Killers). During gameplay, players' behaviour - actions, chat logs, temporal evolution - will be monitored and analysed offline to identify 
them into an stereotype. Actions and states will be tagged in order to influence both the behaviour of NPCs and the way player goals should be completed. Each Character Agent's planner will identify the appropriate actions to fulfill a given landmark: two characters can fulfill the same landmark in different ways, creating the illusion of personality.

\subsection{Adding interactivity to narrative}

Interactivity is achieved by giving enough choices to the player to give an illusion of free will. The actions planned by the Character Agents representing actual players will be enforced in the form of missions or quests offered to the correspondent player taking into account both the IC and the OOC roles. The player, however, will be free to choose; if the player gets too far from the story line, its Character Agent can negotiate (as seen above) changes to the environment to keep the action in the boundaries of the storyline.

However, if the OOC role of a player's Character Agent predicts that the story plans incoming from the Plot Agent are not feasible or too incompatible with the individual plan, framing operators will be checked and studied, and there will be a negotiation process with the Plot Agent to propose and apply them, resulting in applications of making

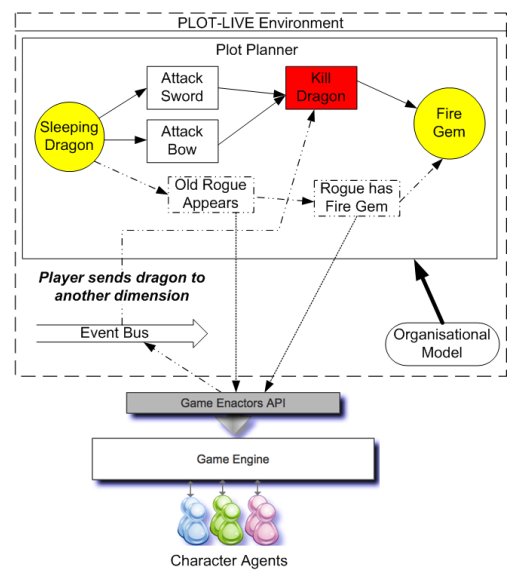

Fig. 4. System Architecture and usage of late commitment and make events happen events happen or late commitment.

In the example shown in Figure 4 , the player is supposed to kill the dragon (either with a sword or a bow) in order to obtain the fire gem from it. However, instead of performing the attack, the player decides to cast a spell on the dragon, sending it to another dimension. As the dragon and the player are in different dimensions, the player cannot obtain the fire gem, and thus, the plot cannot advance. The Plot Agent is able to recover the plot from this deviation by: 1) receiving the event that the player has sent the dragon to another dimension, 2) reacting by introducing an event (make events happen) on the game via the game enactor, e.g., an old rogue appears, as he was hiding in the shadows of the dragon cave, and 3) introducing a late commitment in the plot, via the game enactor, e.g. it comes out that the old rogue has the fire gem, as he had stolen it from the dragon before, and decides to give it to the player. 


\section{Conclusions}

In this paper we have proposed an adaptation of an already existing organisational framework for games for its use in scenarios where the narrative paradox can be tested. The purpose is two-fold. First, we want to test storyline dynamic adaptation in CONCIENS applied to free-roaming games such as multiplayer roleplaying games. Second, we want to explore if emergent narrative can improve with the use of organisational models, strongly focusing on the compromise between character freedom and plot design.

We use CONCIENS as a sandbox for applying the research of our agents group. By combining emergent narrative to the framework, we want to do research not only on narrative in itself but also applied to social aspects, both in-game (NPCs behaving as part of a society), and out-of-game (studying the interaction between players and between a player and the NPCs), from different perspectives: organisational, normative, emotion representation and detection, user profiling, gamification, and so on.

In order to provide empirical results, we have already connected CONCIENS to an open-source World of Warcraft server. Our immediate plan is to test the dynamic generation of missions by using the techniques described in Section 4.

\section{References}

1. Aldewereld, H., Padget, J., Vasconcelos, W., Vázquez-Salceda, J., Sergeant, P., Staikopoulos, A.: Adaptable, Organization-Aware, Service-Oriented Computing. Intelligent Systems ( 25(4), 80-84 2010)

2. Alvarez-Napagao, S., Gómez-Sebastià, I., Vázquez-Salceda, J., Koch, F.: cOncienS: Organizational Awareness in Real-Time Strategy Games. Proc. of the 13th Int. Conf. of the Catalan Assoc. for Artificial Intelligence, IOS Press ( 220, 69-78 2010)

3. Alvarez-Napagao, S., Koch, F., Gómez-Sebastià, I., Vázquez-Salceda, J.: Making games ALIVE: an organisational approach. Agents for Games and Simulations II, Springer-Verlag ( LNAI 6525, 112-124 2010)

4. Arinbjarnar, M., Kudenko, D.: Duality of Actor and Character Goals in Virtual Drama. Intelligent Virtual Agents, Springer-Verlag ( LNAI 5773, 386-392 2009)

5. Aylett, R.: Narrative in Virtual Environments - Towards Emergent Narrative. AAAI Narrative Intelligence Symposium ( FS-99-01, 83-86 1999)

6. Aylett, R., Louchart, S., Dias, J., Paiva, A., Vala, M.: FearNot! - An Experiment in Emergent Narrative. Intelligent Virtual Agents, Springer-Verlag ( LNAI 3661, 305-316 2005)

7. Bartle, R.: Designing Virtual Worlds. (New Riders Games 2003)

8. Bateman, C., Boon, R.: 21st Century Game Design. (Charles River Media 2005)

9. Louchart, S., Aylett, R.: Building Synthetic Actors for Interactive Dramas. AAAI Fall Symposium on Intelligent Narrative Technologies ((FS-07-05), 63-71 2007)

10. Nareyek, A.: Game AI Is Dead. Long Live Game AI! (Intelligent Systems 2007)

11. Swartjes, I.: Whose story is it anyway? How improv informs agency and authorship of emergent narrative. (PhD Thesis, University of Twente 2010)

12. Westra, J., van Hasselt, H., Dignum, V., Dignum, F.: On-line Adapting Games using Agent Organizations. IEEE Symposium on Computational Intelligence and Games (CIG'08) (pp 243-250 2008) 